\title{
Tunneling rate fluctuations induced by nonlinear resonances: A quantitative treatment based on semiclassical arguments
}

\author{
Luca Bonci, ${ }^{1}$ Andrea Farusi, ${ }^{2}$ Paolo Grigolini, ${ }^{1,2,3}$ and Roberto Roncaglia ${ }^{4}$ \\ ${ }^{1}$ Center for Nonlinear Science, University of North Texas, P.O. Box 305370, Denton, Texas 76203 \\ ${ }^{2}$ Dipartimento di Fisica dell'Università di Pisa, Piazza Torricelli 2, 56127 Pisa, Italy \\ ${ }^{3}$ Istituto di Biofisica del Consiglio Nazionale delle Ricerche, Via San Lorenzo 26, 56127 Pisa, Italy \\ ${ }^{4}$ Foundation for Research and Technology-Hellas, Institute of Electronic Structure and Laser, P.O. Box 1527, \\ 71110 Heraklion, Crete, Greece \\ (Received 3 December 1997; revised manuscript received 26 June 1998)
}

\begin{abstract}
We investigate the tunneling process between two symmetric stable islands of a forced pendulum Hamiltonian in the weak chaos regime. We show that when the tunneling doublet is quantized over a classical nonlinear resonance the tunneling rate strongly deviates from the semiclassical prediction. This mechanism is responsible for the irregular dependence of the tunneling rate on the system parameters. The weak-chaos condition allows us to make a theoretical prediction that agrees very well with the numerical results. This opens up a possible avenue to a general theory on the dependence of quantum tunneling on classical chaos.
\end{abstract} [S1063-651X(98)06511-8]

PACS number(s): 05.45.+b, 03.65.Sq, 73.40.Gk

One of the most attractive aspects of classically chaotic quantum systems is the surprising behavior of the tunneling rate. This is shown to be an erratic function of the system parameters [1-4]. This means that the tunneling rate undergoes fluctuations that enhance, or reduce, its intensity by several orders of magnitude, compared to the smooth and regular behavior corresponding to the traditional conditions.

A kind of general agreement has been reached by the researchers working on this hot issue. This is that the tunneling properties must be traced back to the crossing of the tunneling doublet with a third state [5], which corresponds to the chaotic region of the classical phase space [2,3]. We shall be referring to these phenomena as chaos assisted tunneling (CAT) processes, since this is the definition generally adopted in the literature in spite of the fact that the influence of chaos can also reduce the rate of the tunnel process. It is also thought, at the level of merely qualitative arguments, that the erraticlike behavior of the tunneling rate depends on the chaotic nature of the third state. It has to be pointed out that a semiclassical treatment represents a natural way of establishing a connection between the quantum properties, tunneling rate in the case under discussion, and the classical phase space. Unfortunately, these methods cannot be applied to the chaotic states in the standard Einstein-Brillouin-Keller (EBK) form [6], and this is probably the reason why all the results reached so far on this interesting issue fail to supplement the qualitative arguments with precise quantitative predictions. Recently Doron and Frischat [7] have addressed this interesting issue by means of nonordinary semiclassical techniques.

This paper is devoted to describing the discovery that the processes observed in the literature so far, the CAT processes, are a special case of a more general phenomenon. The third state invoked by all the researchers of this field need not be chaotic. Here we show that the same qualitative behavior as that observed in [1-3] becomes ostensible also as an effect of the crossing with regular states. In this case the crossings of states, and thus the fluctuation of the tunneling doublets, can be directly related to the classical phase- space structure and in particular to the destruction of the regular tori by the nonlinear resonances. This makes it possible for us to derive a quantitative prediction, which turns out to agree remarkably well with the numerical results. Note that the main difference with the approach adopted by Doron and Frischat [7] is that these authors study a case of fully developed chaos, a physical condition that forces them to depart from the ordinary semiclassical methods. Here, on the contrary, we focus on the onset of chaos, a condition making it easier for us to establish a connection between quantum tunneling and classical dynamics.

To address the problem of the connection between tunneling and classical phase-space structure we consider the following Hamiltonian:

$$
H=\frac{p^{2}}{2}+v_{1} \cos (2 q)+v_{2} \cos (2 q-\Omega t) .
$$

This is a forced system whose dynamical properties are well known. Depending on the value of the perturbation strength $v_{2}$, the phase space can show either regular or chaotic dynamics. For $v_{2}=0$ this is nothing but an ordinary, and integrable, pendulum. Increasing the value of $v_{2}$ makes the dynamics nonintegrable and eventually chaotic. Figure 1 shows the effect of setting $v_{2}=0.005$. We can easily identify the isolated resonances of order $1 / 8,1 / 7$, and $1 / 6$, resulting from the destruction of tori with winding numbers rationally connected to the perturbation frequency. Notice also the stochastic layer close to the separatrix, where the higher-order resonances overlap, giving rise to fully developed chaos.

To study the quantum dynamics of this system, and in particular its tunneling properties, we set periodic conditions at the borders of the interval $q \in[0,2 \pi]$. This has the effect of creating an enlarged phase space consisting of two identical cells. The quantum-mechanical eigenstates must be either symmetric or antisymmetric with respect to a $\pi$-translation along the $q$ axis, and some eigenstates exist whose linear combination is essentially located in only one of the two cells. According to [8] the time evolution is described by means of a unitary operator, which evolves the 


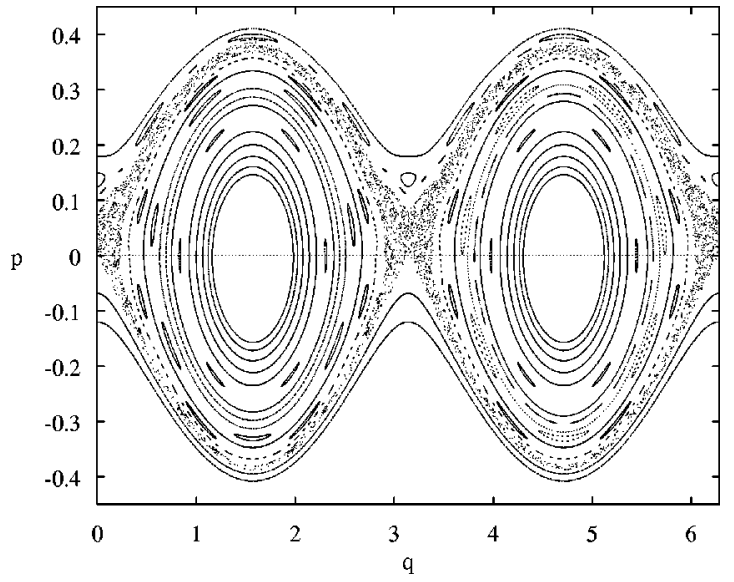

FIG. 1. Stroboscopic plot of the classical dynamics driven by Hamiltonian (1). The values of the system parameters are $\Omega=2$, $v_{1}=0.035, v_{2}=0.005$. Some nonlinear resonances of order $1 / n$ are visible: those below the separatrix refer to $n=6,7$, and 8 , and that above it to $n=8$.

quantum state by one entire period of the external perturbation. This is called the Floquet operator and it is denoted by $\hat{F}$. The generalized eigenstates $u_{n}(q, t)$ and eigenvalues $\varepsilon_{n}$ of $\hat{F}$ are derived from the eigenvalue equation:

$$
\left(H-i \hbar \frac{\partial}{\partial t}\right) u_{n}(q, t)=\varepsilon_{n} u_{n}(q, t),
$$

where $u_{n}(q, t)=\exp \left(-i \phi_{n} t\right) \psi_{\phi_{n}}(q, t)$ is by construction time periodic, $u_{n}(q, t)=u_{n}(q, t+T)$, and $\varepsilon_{n}$ is defined by $\varepsilon_{n}$ $\equiv \hbar \phi_{n}$. One important feature of the Floquet formalism is the Brillouin-zone structure of the spectrum: every eigenstate $u_{n}(q, t)$ results in the wider class of eigenstates and eigenvalues defined, respectively, by

$$
u_{n, m}(q, t) \equiv u_{n}(q, t) e^{i m \Omega t}
$$

and

$$
\varepsilon_{n, m} \equiv \varepsilon_{n}+\hbar m \Omega,
$$

where $m \in Z$. It is evident that the functions belonging to the same class represent the same solution to Eq. (2). Therefore, if an eigenstate has a given eigenvalue $\varepsilon$, which does not belong to the first Brillouin region, $0 \leqslant \varepsilon<\hbar \Omega$, it is convenient to fold it back to this region by means of the prescription (4). To avoid confusion we denote the resulting energies with the symbol $E$.

The eigenstates $u_{n, m}(q, t)$ reflect the translational invariance $q \rightarrow q+\pi$ and can be labeled as odd or even with respect to this symmetry operation. Thus the energy spectrum of the Floquet operator appears as a series of doublets $E_{n, \pm}$ with \pm indicating the translation symmetry. The rate of the tunneling process is determined by the energy splittings $\Delta E_{n}=\left|E_{n,+}-E_{n,-}\right|$, which, in turn, determine the dynamics of a generic wave packet initially located in one of the two islands. For simplicity here we focus on the energy splitting of the "main doublet," which represents the natural extension of the fundamental-state doublet of an autonomous Hamiltonian to the Floquet picture. This is defined as the set of the two eigenstates of the Floquet operator that have the largest overlap with a minimum-uncertainty state located at the center of one of the two stable islands [4].

We adopt the following numerical procedure. The Floquet matrix $\hat{F}$ is determined by the numerical integration of Eq. (2) and the eigenvalues and the eigenstates are subsequently obtained by numerical diagonalization. The results for the main-doublet splitting $\Delta E \equiv \Delta E_{0}$, as a function of $v_{1}$ are shown in Fig. 2(a) for different values of the perturbation parameter $v_{2}$. We see that accordingly with the semiclassical prediction [9], in the unperturbed case $\Delta E$ is a smooth function of $v_{1}$. However, when $v_{2}$ is given an even small but finite value, $\Delta E$ strongly departs from the smooth behavior, with an increase, or decrease, by several orders of magnitude. This behavior is similar to that of $[2,3]$, where the splitting irregularity has been related to the crossings of the tunneling doublet with a third level, which belongs to the chaotic region of the corresponding classical system. To confirm the connection of these results with the third-state theory, we calculate the first energy levels $E_{n}$. In Fig. 2(b) we show those of them that cross the main doublet in the same $v_{1}$ interval as that of Fig. 2(a). We see clearly that the splitting irregularities correspond to the crossing between the main doublet and a third state, or more precisely, a second doublet [5]. The effect of level crossing with a weakly perturbed Hamiltonian is well established: the spectrum is modified only in the vicinity of the crossing that becomes an avoided level crossing, thereby resulting in significant changes of the main-doublet levels, and thus of the splitting $\Delta E$. Note that the avoided crossing is too small to be visible in the scale of Fig. 2(b).

Is this effect a CAT process? Do the states colliding with the main doublet belong to the chaotic region of the phase space? The answer is incontrovertible: in general they do not. This is so because, at least for the smallest values of $v_{2}$ used in Fig. 2(a), the doublets 5, 6, and 7 belong to the regular region. In fact $v_{2}=0.0001$, a value at which the fluctuations of the tunneling rate are already very strong, means a perturbation weaker than that used to derive Fig. 1: the corresponding phase space is even more regular, and thus barely distinguishable from the unperturbed one except possibly for a thin stochastic layer around the separatrix. We think, therefore, that the results of Fig. 2(a), with the help of Fig. 1, afford a compelling numerical evidence that the tunneling rate can be characterized by strong fluctuations without necessarily involving the interaction with a strongly chaotic region. The splitting irregularities are shown below to be caused by the birth, in the regular phase-space region, of nonlinear resonances, a fact that, surprisingly enough, has been overlooked by the literature on this field of research.

Note that further progress on the CAT processes has been hampered by a major difficulty: it is not yet known how to realize a proper semiclassical picture of the chaotic states and, consequently, how to evaluate the tunneling matrix elements. The crossings 5, 6, and 7 of Fig. 2(a), on the contrary, are compatible with the semiclassical quantization of Hamiltonian (1), along the lines established by Breuer and Holthaus [10] and by Bohigas et al. [2]. The analysis here is restricted to the crossing between the main doublet and the states lying below the separatrix. 


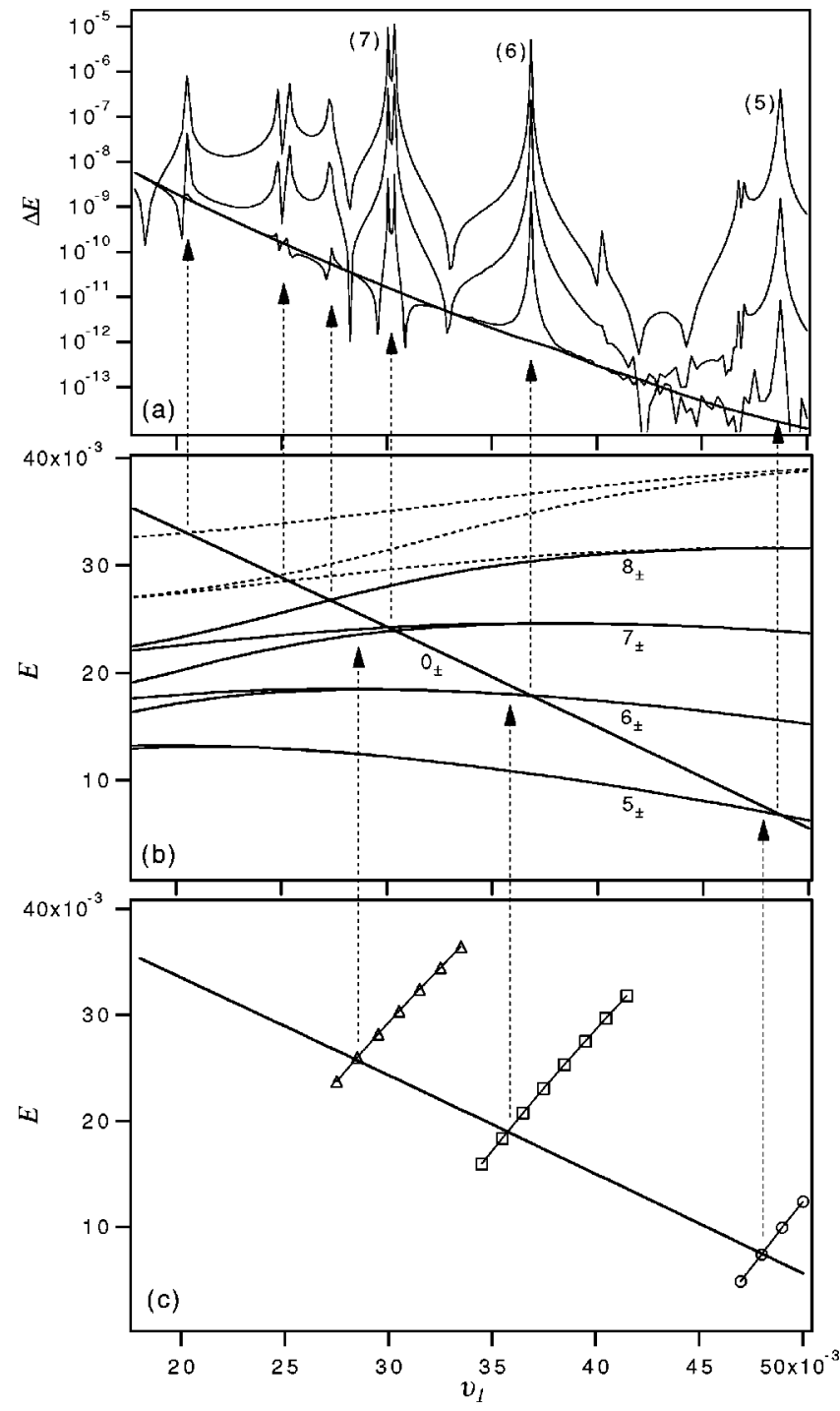

FIG. 2. The origin of the tunneling rate fluctuations for Hamiltonian (1) with $\Omega=2$ and $\hbar=0.025$. (a) The main doublet splitting $\Delta E$ as a function of $v_{1}$. The thick full line denotes the unperturbed case $\left(v_{2}=0\right)$. The other curves, from the bottom to the top, refer to $v_{2}=0.0001,0.001$, and 0.005. (b) The levels $E_{n}$ as functions of $v_{1}$. The full line labeled by $0 \pm$ denotes the main doublet. The other levels are indicated by either full or dashed lines according to whether their crossing with the main doublet occurs below or above the separatrix. The full lines are labeled by their quantum numbers. The value of the perturbation strength is $v_{2}=0.001$. All the energy splittings, also at the avoided crossings, are not visible on this scale. (c) The energy of the fundamental doublet (full line) and the energies of the renormalized quantum resonances as solutions of Eq. (7) (symbols). The resonances are of order $1 / n=1 / 5$ (circles), $1 / 6$ (squares), and 1/7 (triangles). The vertical arrows are guides to the eye connecting the theoretical crossings of (c) to the numerical crossings of (b), and these to the tunneling peaks of (a).

We proceed as follows. Adopting a perturbative approach, we replace $H$ with $H_{0}=p^{2} / 2+v_{1} \cos (2 q)$, and write the condition of level crossing in the Brillouin zone as [10]

$$
H_{0}\left(\hbar\left(n+\frac{1}{2}\right)\right)+\hbar \Omega m=H_{0}\left(\hbar\left(n^{\prime}+\frac{1}{2}\right)\right)+\hbar \Omega m^{\prime} .
$$

Let us assume $\hbar$ to be so small as to make it possible to consider $\hbar\left(n-n^{\prime}\right)$ to be a small expansion parameter. By using $J=\hbar(n+1 / 2)$, we obtain

$$
\bar{\omega}=\frac{\Delta m}{\Delta n} \Omega+O(\hbar),
$$

where $\bar{\omega} \equiv \bar{\omega}(J)=\partial H_{0} / \partial J$ is the frequency of the unperturbed libration as a function of the classical action $J, \Delta n$ $\equiv n^{\prime}-n$ and $\Delta m \equiv m-m^{\prime}$. Equation (6) corresponds to the classical condition for the onset of nonlinear resonances. This means that, for sufficiently small $\hbar$ 's, the crossing of the two unperturbed levels $E_{n, m}^{0}$ and $E_{n^{\prime}, m^{\prime}}^{0}$ occurs as a quantum reflection of the birth of a nonlinear resonance of order $\Delta m / \Delta n$ in the classical phase space. The latter has the same energy as the torus determining the semiclassical quantization of one of the two crossing levels. On the basis of this result the level crossing concerning the main doublet ( $n$ $=0)$ corresponds to the overlap between the semiclassical quantization torus of the fundamental state and a nonlinear resonance of the appropriate order. Notice that, in principle, Eq. (6) involves resonances of any order, thereby making a given level undergo a virtually infinite number of crossings upon change of a system parameter. However, Ref. [10] shows that the perturbation strength at the crossing is determined by the order of the corresponding nonlinear resonance, and that the first-order resonances $(\Delta m=1)$ produce the most intense effects.

The theoretical result of Eq. (6) implies such a strong semiclassical condition as to make it difficult to check it numerically. To bypass this limitation we apply to Eq. (5) the second-order approximation. Thus we obtain

$$
\bar{\omega}=\frac{\Delta m}{\Delta n} \frac{\Omega}{[1+(\hbar / 2)(d \bar{\omega} / d E) \Delta n)]}+O\left(\hbar^{2}\right),
$$

where $d \bar{\omega} / d E$ can be expressed analytically via $\bar{\omega}$ $=\pi \sqrt{v_{1}} / K(k), K(k)$ is the elliptic function, and $k^{2}=(E$ $\left.+v_{1}\right) / 2 v_{1}$. Equation (7) looks like Eq. (6) with the frequency $\Omega$ properly renormalized and thus depending on $\hbar$, as well as on the energy of the colliding level.

This theoretical prediction on the quantum crossings is illustrated by Fig. 2(c). We note that the fundamental doublet energy is denoted by a single full line because, as in Fig. 2(b), the energy splitting is not visible in this scale. The prediction stemming from Eq. (7) must be compared to the numerical quantum crossings of Fig. 2(b), which occur both above and below the separatrix. Here we see that the accuracy of the prediction is good and, as expected, becomes better with resonances of smaller order. A still better agreement is expected from smaller values of $\hbar$.

We now invite the reader to compare Figs. 2(b) and 2(a), while keeping in mind their earlier correspondence with Fig. 2(c). This results in a vivid explanation of the "fluctuations" of the tunneling rate. This is made especially evident by a thorough examination moving from smaller to larger values of the perturbation strength $v_{2}$. We see that at $v_{2}=0.0001$ the deviations from the unperturbed behavior are significant only in the close vicinity of the level crossings, thus lending support to our perturbative approach. With the increase of 
$v_{2}$, the peaks broaden and overlap one another and for still larger values of $v_{2}$ new peaks come forth corresponding to crossings of higher order.

The peaks around $v_{1}=0.025$ and $v_{1}=0.0275$ refer to levels close to the separatrix, which at properly large $v$ 's are embedded in the chaotic region. The peak at about $v_{1}$ $=0.020$ refers to an above-separatrix level. As a consequence of this, the dynamical classical process behind these three peaks is compatible with the motion from one to the other potential well. For these reasons one would be tempted to conclude that the corresponding transition rates are the largest. We see, on the contrary, that the peaks referring to a below-separatrix level crossing $\left(v_{2}>0.030\right)$ are more intense than the peaks due to the above-separatrix level crossings. We also note that at the highest value of $v_{2}$ used in Fig. 2(a), when the tunneling rate results in the maximum departure from the unperturbed prediction, the classical phase-space structure is still almost regular, as shown by Fig. 1, which refers to the same value of $v_{2}$.

In conclusion, we have identified a process resting on the role of isolated nonlinear resonances rather than that of the connected chaotic sea. The corresponding tunneling rate $\Delta E$ exhibits fluctuations that can be even more intense than those provoked by the crossing with chaotic states. This can be accounted for by using the same heuristic arguments as those adopted in Refs. [2,3]. These authors argue that the intensity of the process is proportional to the matrix element $V$ connecting one of the partners of the tunneling doublet, state $|r\rangle$, to the crossing state $|c\rangle$. This crucial parameter is roughly proportional to the overlap between $|c\rangle$ and $|r\rangle$. We can predict its value with heuristic arguments based on the observation of the Husimi distribution of the eigenstates (not shown here). The ground state is represented by almost Gaussian packets located at the centers of the regular islands, the regular crossing state corresponds to a double ringshaped bun surrounding the Gaussian packets, and the chaotic state to an 8-shaped distribution lying on the chaotic region of Fig. 1. As the value of $\hbar$ decreases, the Gaussian state shrinks, the regular distribution collapses on its quantization torus, and the chaotic state remains localized in the chaotic layer. This produces a decrease of the overlap among different states, and consequently an overall decrease of the tunneling rate. However, a mere inspection of Fig. 1 leads to the incontrovertible conclusion that the effect of the regularregular crossing is expected to remain larger than that of the regular-chaotic one. This is made compelling by the topology of the phase space: the overlap with the closer regular state remains larger than the overlap with the farther chaotic one.

We note also that the values of the parameter $v_{1}$ corresponding to the tunneling-rate peaks appear to be independent of the intensity of the perturbation strength $v_{2}$. Should this independence remain unaffected by the emergence of fully developed chaos, the results of this paper would shed light into the physics of the CAT processes. The assessment of this key property requires further research work and the adoptions of different parameter values. At the present stage we are forced to limit ourselves to making conjectures. Our conjecture is that the position of the peaks remains unchanged even when the overlap of the isolated resonances occurs, and a condition of full chaos develops. This changed condition produces the broadening of these peaks, the birth of higher-order crossings and the resulting merging of all of them into a single erraticlike structure, without influencing their original position. Thus we think that even after the crossover to the chaotic regime, significant signs of the peaks originated by the quasiintegrable nonlinear resonances remain.

Before concluding, we want to point out that these results are qualitatively similar to those obtained in Ref. [4]. These physical effects, as here shown, can be accounted for very well, both qualitatively and quantitatively. This means that the theoretical arguments of this paper provide an exhaustive account for the "plateau effect" of [4]. This is an important task that has recently challenged the efforts of some groups (see Ref. [11]). Should the phenomenon illustrated in [4] be proved to be a form of CAT, this would be a strong support of our conviction that the semiclassical arguments of this paper are a significant step ahead towards a quantitative prediction of CAT processes.

R.R. thanks the CEE for Programme Research Training (TMR) Grant No. ERB4001GT952681, while L.B. acknowledges the Italian CNR and NATO for financial support.
[1] W. A. Lin and L. E. Ballentine, Phys. Rev. Lett. 65, 2927 (1990); F. Grossmann, T. Dittrich, P. Jung, and P. Hänggi, ibid. 67, 516 (1991).

[2] O. Bohigas, S. Tomsovic, and D. Ullmo, Phys. Rep. 223, 43 (1993).

[3] S. Tomsovic and D. Ullmo, Phys. Rev. E 50, 145 (1994).

[4] R. Roncaglia, L. Bonci, F. Izrailev, Bruce J. West, and P. Grigolini, Phys. Rev. Lett. 73, 802 (1994).

[5] The expression "crossing with a third state"' is borrowed from the literature, but it is probably inappropriate, since the third state can also consist of a doublet with a so small energy separation as to prevent the possibility of detecting a crossing at a time.
[6] A. Einstein, Verh. Dtsch. Phys. Ges. 19, 82 (1917); L. Brillouin, J. Phys. Radium 7, 353 (1926); J. B. Keller, Ann. Phys. (N.Y.) 4, 180 (1958).

[7] E. Doron and S. D. Frischat, Phys. Rev. Lett. 75, 3661 (1995); S. D. Frischat and E. Doron, Phys. Rev. E 57, 1421 (1998).

[8] Ya. B. Zel'dovich, Sov. Phys. JETP 24, 1006 (1967); J. H. Shirley, Phys. Rev. 138, B979 (1965).

[9] M. Wilkinson, Physica D 21, 341 (1986).

[10] H. P. Breuer and M. Holthaus, Ann. Phys. (N.Y.) 211, 249 (1991).

[11] E. M. Zanardi, J. Gutiérrez, and J. M. Gomez Llorente, Phys. Rev. E 52, 4736 (1995). 